\title{
Alpha Fetoprotein L3 Measurement
}

National Cancer Institute

\section{Source}

National Cancer Institute. Alpha Fetoprotein L3 Measurement. NCI Thesaurus. Code C96564.

The determination of the amount of alpha fetoprotein $L 3$ present in a sample. 